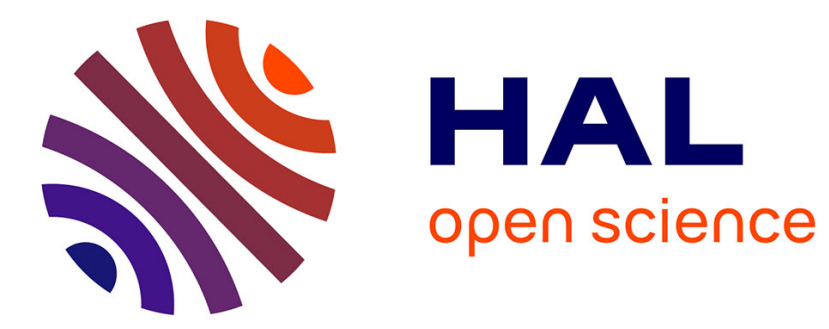

\title{
Optimal Measurement Pose Selection for Joint Stiffness Identification of an Industrial Robot Mounted on a Rail
}

David Guérin, Stéphane Caro, Sébastien Garnier, Alexis Girin

\section{To cite this version:}

David Guérin, Stéphane Caro, Sébastien Garnier, Alexis Girin. Optimal Measurement Pose Selection for Joint Stiffness Identification of an Industrial Robot Mounted on a Rail. IEEE/ASME International Conference on Advanced Intelligent Mechatronics , Jul 2014, Besançon, France. 10.1109/AIM.2014.6878332 . hal-01693092

\section{HAL Id: hal-01693092 https://hal.science/hal-01693092}

Submitted on 3 May 2018

HAL is a multi-disciplinary open access archive for the deposit and dissemination of scientific research documents, whether they are published or not. The documents may come from teaching and research institutions in France or abroad, or from public or private research centers.
L'archive ouverte pluridisciplinaire HAL, est destinée au dépôt et à la diffusion de documents scientifiques de niveau recherche, publiés ou non, émanant des établissements d'enseignement et de recherche français ou étrangers, des laboratoires publics ou privés. 


\title{
Optimal Measurement Pose Selection for Joint Stiffness Identification of an Industrial Robot Mounted on a Rail
}

\author{
David Guérin, Stéphane Caro, Sébastien Garnier, Alexis Girin
}

\begin{abstract}
The paper focuses on the identification of elastostatic properties of an industrial serial robot mounted on a rail. It proposes an identification procedure in order to find the optimal robot configuration to minimize the impact of measurement errors on the identification accuracy of the stiffness parameters. An experimental setup is designed to perform the identification of all stiffness parameters under industrial conditions. The proposed identification procedure is easy to use and takes little time.
\end{abstract}

\section{INTRODUCTION}

Nowadays, several industrial companies are interested in analysing the possibility to employ serial robots for industrial machining operations. Indeed, serial robots can be more flexible than computer numerical control (CNC) machines and also cost-effective, but, serial robots are usually not as stiff as a CNC machine and they are not designed specifically to perform machining operations.

The IRT Jules Verne launched in 2013 the project ROBOFIN with several industrial partners (DAHER, DCNS, STX France, Loiretech, Multiplast, Europe Technologies and Coriolis Composites) to investigate machining operations on large size pieces performed by a KUKA KR500 L480-2 robot mounted on a 18 meters long rail.

In order to improve machining capabilities of industrial serial robots it is necessary to detect the main factors that are responsible to the robot inaccuracy. Since the introduction of serial robots in industry, several studies have focused on their geometric parameters identification and calibration [1], in order to improve their accuracy. The geometric calibration is mainly focused on the identification of the geometric parameters and joint offsets.

To improve the efficiency of a robot, calibration of non-geometric parameters has to be considered as well. Identification and calibration of robot stiffness parameters (elasto-static calibration) is essential too for a serial machining robot [2]. When a serial robot performs machining operations, the efforts applied on the robot end-effector cause deformations on the robot components, reducing the quality of the machined parts. Generally, for an industrial serial robot the compliance of the bodies is assumed to be negligible with respect to the joint compliance [3], [4]

This work is supported by the Institut de Recherche Technologique Jules Verne, Bouguenais, France (Project ROBOFIN, ROBOtique de FINinition)

D. Guérin and A. Girin are with Robotics Team, IRT Jules Verne, Chemin du Chaffault, 44340 Bouguenais, France \{david.guerin, alexis.girin\}@irt-jules-verne.fr

S. Caro and S. Garnier are with the Institut de Recherche en Communication et Cybernétique de Nantes, UMR CNRS n6597, 1 rue de la Noë, 44321 Nantes, France \{stephane.caro, sebastien.garnier\}@irccyn.ec-nantes.fr

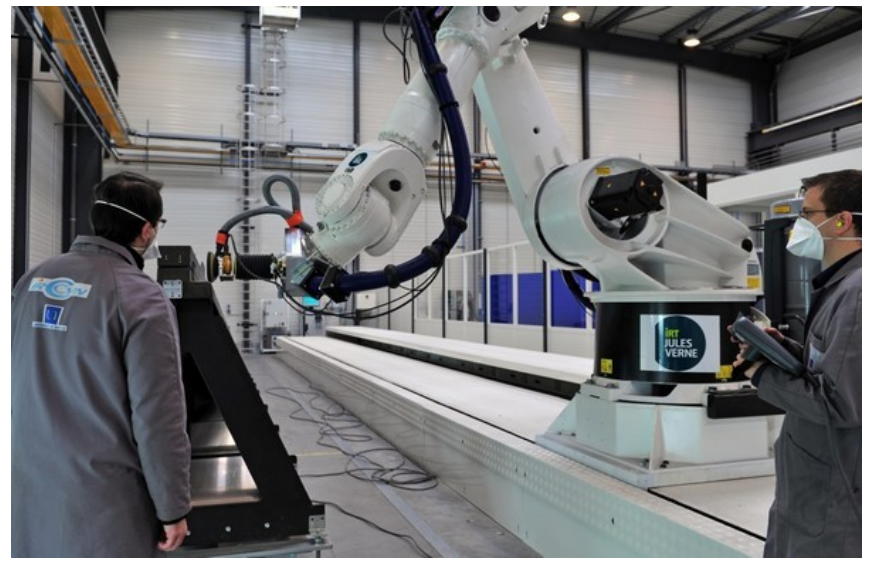

Fig. 1. KR500 L480-2 MT mounted on rail located at IRT Jules Verne

and [5]. Nowadays, there are still many research works focusing on the robot stiffness identification. Gautier et al. [6] proposed an off-line joint stiffness identification method based on motor torque output data. In Milutinovic et al. [7] the stiffness identification is applied to a 5-axis serial robot used to perform machining operations, in order to analyse the impact of each joint's compliance on the robot stiffness. In [8], a stiffness identification was performed to a Mitsubishi PA10-6CE, in order to improve static positioning accuracy of the robot.

During machining operations, the robot can be subjected to chattering. Pan et al. performed some studies about the elasto-dynamic identification of serial robots. Their research work aimed at avoiding chatter by selecting the optimal cutting parameters [9]. Other non-geometric parameters can affect robot efficiency. Gong et al. [10] studied the impact of thermal effects on serial robot deformations.

In industrial environment, robot parameters identification may be time consuming. Hence, some works have focused on the optimisation of measurement configurations [11], [12]. The study described in [13] and [14] is focused on the optimisation of the measurement configurations for robot stiffness identification. In this paper, a method is proposed to decrease the time required for the stiffness identification experiments by providing a simple method easy to use and by minimising the number of measurements configuration. Moreover, this method has been developed, to improve the identification accuracy and is based on the work of Wu et al. [15] and has been adapted to a serial robot mounted on a rail. This paper also presents an experimental setup used to identify all stiffness parameters. Section II presents the track 


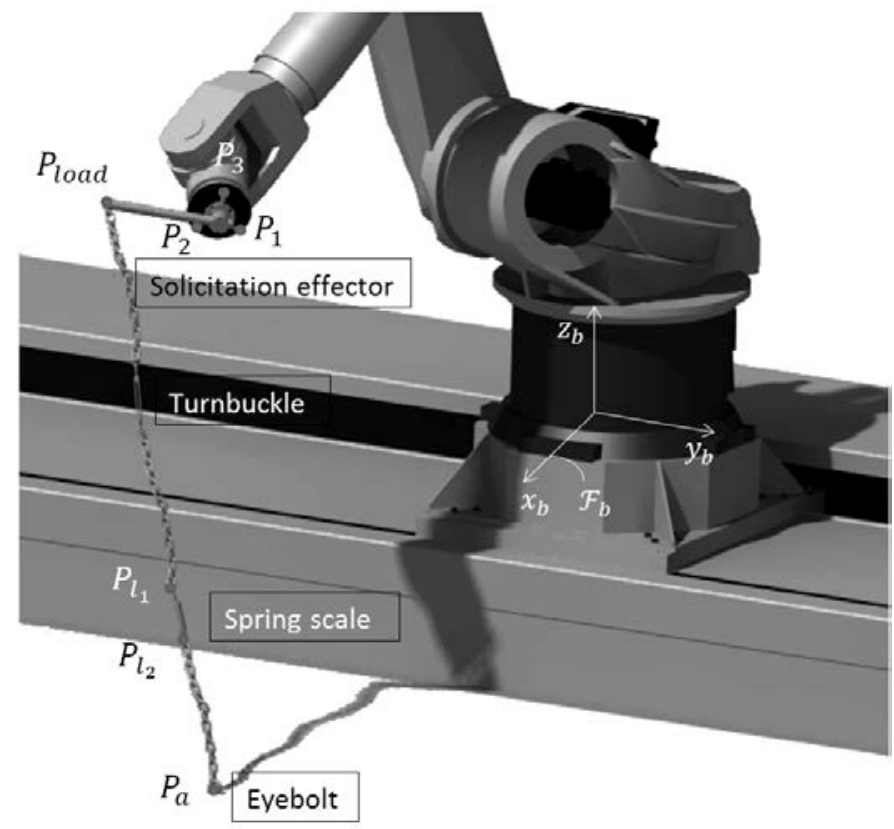

Fig. 2. Experimental setup used to exert an external wrench on the robot end-effector

mounted robot and measurement equipment. Section III deals with the kinematic modelling and stiffness modelling of the robot. Section IV details the method used to determine the optimal measurement configurations. Section V provides simulation results. Section VI deals with the conclusion and the future work.

\section{ROBOTIC CELL AND EXPERIMENTAL SETUP}

The presented work aims to investigate machining operations performed with a serial robot mounted on a rail. The following case study has been taken into consideration. The robotic machining cell located at the IRT Jules Verne and shown in Fig. 1 is composed of a KUKA KR500 L480-2 MT robot and a rail. The robot rated payload is equal to $480 \mathrm{~kg}$. The rail is $18 \mathrm{~m}$ long and has been developed by Sud-Ouest Système company. In what remains, the KUKA KR500 L480-2 MT robot will be referred to as KR500. In order to identify the stiffness parameters of the KR500 and the rail, a solicitation effector developed at IRCCyN is mounted on the KR500 end-effector. Moreover a chain with an embedded turnbuckle is attached to the solicitation effector at one end and to the floor at the other end, namely, at point $P_{a}=\left[x_{p_{a}} y_{p_{a}} z_{p_{a}}\right]$, as illustrated in Fig. 2 .

The aim of this experimental setup is to be cost effective with no extra infrastructure and easy to integrated in industrial environment. Moreover this setup can be used to identify all stiffness parameters of the presented robotic cell.

An identification procedure will be developed in Section IV in order to find the robot configurations associated with the optimal measurement poses and the optimal location of the attachment point, $P_{a}$, such that the effect of measurement errors on the identification accuracy of the stiffness parameters is a minimum. In this identification procedure, the

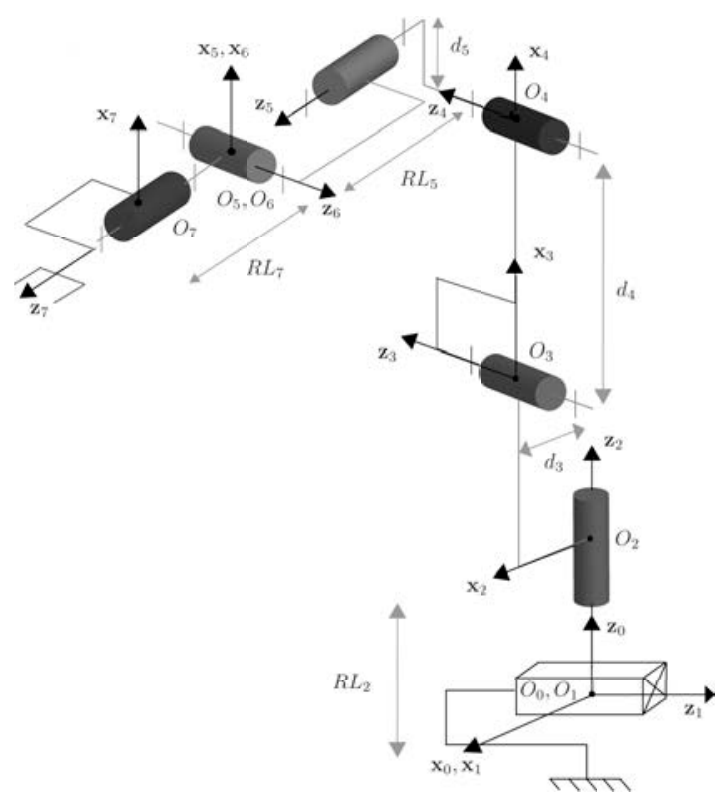

Fig. 3. Kinematic drawing of the KR500 L480-2 MT mounted on a rail

TABLE I

MDH PARAMETERS OF THE KR500 ROBOT MOUNTED ON A RAIL

\begin{tabular}{ccccc}
\hline \hline$j$ & $\alpha_{j}[\mathrm{rad}]$ & $d_{j}[\mathrm{~mm}]$ & $\theta_{j}[\mathrm{rad}]$ & $r_{j}[\mathrm{~mm}]$ \\
\hline 1 & $-\pi / 2$ & 0 & 0 & $r_{1}$ \\
2 & $\pi / 2$ & 0 & $\theta_{2}$ & 1048 \\
3 & $\pi / 2$ & 500 & $\theta_{3}$ & 0 \\
4 & 0 & 1300 & $\theta_{4}$ & 0 \\
5 & $\pi / 2$ & 55 & $\theta_{5}$ & 1525 \\
6 & $-\pi / 2$ & 0 & $\theta_{6}$ & 0 \\
7 & $\pi / 2$ & 0 & $\theta_{7}$ & 290 \\
\hline \hline
\end{tabular}

Cartesian coordinates of points $P_{1}, P_{2}, P_{3}, P_{l_{1}}$ and $P_{l_{2}}$ will be measured by a Leica Laser Tracker and retroreflectors.

\section{ROBOT STIFFNESS MODELLING}

\section{A. Kinematic modelling and parametrization}

Fig.3 illustrates a kinematic drawing of the KR500 mounted on a rail. The modified Denavit Hartenberg $(\mathrm{mDH})$ parameters are used to parametrize the KR500 robot and the rail.

- the $\mathbf{z}_{j}$ axis is along the axis of joint $j$

- the $\mathbf{x}_{j}$ axis is aligned with the common normal between $\mathbf{z}_{j}$ and $\mathbf{z}_{j+1}$.

- the origin $O_{j}$ is the intersection point of $\mathbf{z}_{j}$ and $\mathbf{x}_{j}$

The $\mathrm{mDH}$ parameters of the KR500 mounted on a rail are given in Table I.

The direct kinematic model gives us the relation between the end-effector twist, $\mathbf{t}$, and the joint rates vector $\dot{\mathbf{q}}$, expressed in the base frame $\mathcal{F}_{b}$ :

$$
\mathbf{t}=\mathbf{J}(\mathbf{q}) \dot{\mathbf{q}}
$$

where $\mathbf{q}=\left[\begin{array}{lllllll}r_{1} & \theta_{2} & \theta_{3} & \theta_{4} & \theta_{5} & \theta_{6} & \theta_{7}\end{array}\right]^{T}$ is the joint coordinate vector, $\dot{\mathbf{q}}=\left[\begin{array}{lllllll}\dot{r}_{1} & \dot{\theta_{2}} & \dot{\theta}_{3} & \dot{\theta}_{4} & \dot{\theta}_{5} & \dot{\theta}_{6} & \dot{\theta}_{7}\end{array}\right]^{T}$ is the joint velocity 
vector, $r_{1}$ is the prismatic joint length and $\left[\theta_{2} \ldots \theta_{7}\right]$ are the revolutes joint angles. $\mathbf{J}$ is the kinematic Jacobian matrix of the KR500 mounted on a rail.

\section{B. Stiffness model}

Performing machining operations with a serial robot generates an external wrench on the robot end-effector. This external wrench may produce elastic deformations in the robot. The robot links are assumed to be rigid. The elasticity of the rail is modelled by a linear elastic spring along the direction of the rail. The revolute joint stiffness is characterized by a linear elastic torsional spring. The Cartesian stiffness matrix $\mathbf{K}_{X}$ of the KR500 mounted on a rail is expressed as:

$$
\mathbf{K}_{X}=\mathbf{J}^{-T} \mathbf{K}_{q} \mathbf{J}^{-1}
$$

where $\mathbf{K}_{q}$ is the diagonal joint stiffness matrix expressed as:

$$
\mathbf{K}_{q}=\left[\begin{array}{ccccccc}
k_{r_{1}} & 0 & 0 & 0 & 0 & 0 & 0 \\
0 & k_{\theta_{2}} & 0 & 0 & 0 & 0 & 0 \\
0 & 0 & k_{\theta_{3}} & 0 & 0 & 0 & 0 \\
0 & 0 & 0 & k_{\theta_{4}} & 0 & 0 & 0 \\
0 & 0 & 0 & 0 & k_{\theta_{5}} & 0 & 0 \\
0 & 0 & 0 & 0 & 0 & k_{\theta_{6}} & 0 \\
0 & 0 & 0 & 0 & 0 & 0 & k_{\theta_{7}}
\end{array}\right]
$$

Zhang et al. [16] showed that the Cartesian stiffness matrix $\mathbf{K}_{X}$ can be rewritten as follows:

$$
\mathbf{K}_{X}=\mathbf{J}_{l}^{-T} \mathbf{K}_{q} \mathbf{J}_{m}^{-1}
$$

where $\mathbf{J}_{m}$ is the Jacobian matrix expressed at the measurement point and $\mathbf{J}_{l}$ is the Jacobian matrix expressed at the loading point. Therefore, the relationship between the endeffector displacement $\delta \mathbf{d}$ and the external wrench $\mathbf{w}$ can be expressed as:

$$
\delta \mathbf{d}=\mathbf{J}_{m} \mathbf{K}_{q}^{-1} \mathbf{J}_{l}^{T} \mathbf{w}
$$

where $\delta \mathbf{d}=\left[\begin{array}{ll}\delta \mathbf{p} & \phi\end{array}\right]^{T}$ is the vector of the end-effector displacement, with $\delta \mathbf{p}$ and $\phi$ being respectively the pointdisplacement and the rotational error of the robot endeffector. $\mathbf{w}=[\mathbf{f} \mathbf{m}]^{T}$ is the vector of external wrench (forces and moments).

In order to consider the measurement errors and the pointdisplacement of the measurement points Eq. (5) is rewritten as:

$$
\delta \mathbf{p}_{i}=\mathbf{A}_{i} \mathbf{x}+\epsilon_{i} \quad(i=1, \ldots, n)
$$

where $\delta \mathbf{p}_{i}$ is the point-displacement of the $i$ th measurement point, $\mathbf{x}$ is the vector of joint compliances, $\mathbf{x}=$ $\left[1 / k_{r_{1}}, 1 / k_{\theta_{2}}, \ldots, 1 / k_{\theta_{7}}\right]^{T}$ and $\epsilon_{i}$ denotes the measurement error due to the Leica laser tracker. The matrix $\mathbf{A}_{i}$ is composed of the columns of matrix $\mathbf{J}_{l}$ and $\mathbf{J}_{m}$, and the external force $\mathbf{f}$ applied on the loading point, namely,

$$
\mathbf{A}_{i}=\left[\mathbf{J}_{m_{1, i}} \mathbf{J}_{l_{1, i}}^{T} \mathbf{f}_{i}|\ldots| \mathbf{J}_{m_{7, i}} \mathbf{J}_{l_{7, i}}^{T} \mathbf{f}_{i}\right](i=1, \ldots, n)
$$

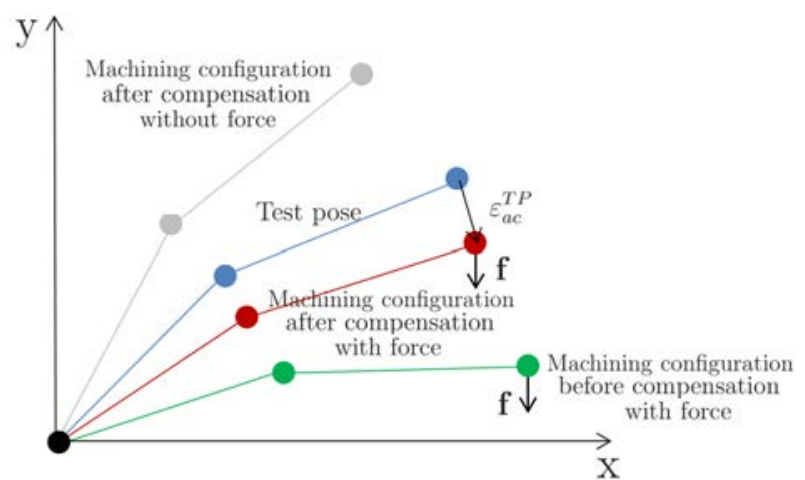

Fig. 4. Drawing representation of the criterion $\varepsilon_{a c}^{T P}$ [17]

\section{OPTIMAL STIFFNESS IDENTIFICATION FOR A TRACK MOUNTED ROBOT}

\section{A. Problem statement}

A method defined in $\mathrm{Wu}$ et al., in 2012 [15] proposed a criterion to obtained optimal measurement poses to identify stiffness parameters of an industrial serial robot. This methodology is focused on the test-pose (TP) base approach. The test-pose is a particular robot configuration in which very good accuracy is required after compensation due to the machining operation to be realized. For experimental validation a vertical wrench was applied on a Kuka KR270-2 robot [17]. In this case, the first joint stiffness cannot be identified, because it is not solicited. Therefore, some other experiments are required for the identification of this first joint stiffness.

In the case of the robot mounted on a rail, an experiment with a vertical wrench leads to similar results for the identification of the first prismatic joint stiffness and the first revolute joint stiffness. Then, the experimental setup presented in Section II is used in order to solicitate all the stiffness of all joints.

Note that the method developed in [15] is used to find the optimal measurement poses for the joint stiffness identification of the KR500 mounted on a rail shown in Fig. 1. However, the optimisation problem at hand has been enhanced in order to find the optimal location of the base attachment point $P_{a}$.

\section{B. Adapted methodology}

The criterion $\varepsilon_{a c}^{T P}$ is employed to evaluate the root-meansquared error of the robot end-effector deflection for a given test pose, as illustrated in Fig 4.

$$
\varepsilon_{a c}^{T P}=\sigma^{2} \operatorname{trace}\left(\mathbf{A}_{0}\left(\sum_{i=1}^{n} \mathbf{A}_{i}^{T} \mathbf{A}_{i}\right)^{-1} \mathbf{A}_{0}^{T}\right)
$$

where the matrix $\mathbf{A}_{0}$ amounts to matrix $\mathbf{A}_{i}$, defined in Eq.(7), evaluated at the test-pose, $\sigma=0.03 \mathrm{~mm}$ is the standard deviation of the measurement noise as used in [17] for the same measurement device. 
TABLE II

CRITERION $\varepsilon_{a c}^{T P}\left[10^{-2} \mathrm{~mm}\right]$, DEFINED IN EQ. (8), AS A FUNCTION OF THE NUMBER OF MEASUREMENT POSES AND THE NUMBER OF EXPERIMENTS, WHILE CONSIDERING THE OPTIMAL LOCATION OF THE BASE ATTACHMENT POINT $P_{a}$

\begin{tabular}{c|cccc}
\hline num of exp & \multicolumn{5}{|c}{ number of measurement poses } \\
& $m_{0}=3$ & $m_{0}=4$ & $m_{0}=6$ & $m_{0}=12$ \\
\hline$n=3$ & 1.980 & & \\
\hline$n=4$ & - & 1.706 & \\
\hline$n=6$ & 1.400 & - & 1.385 \\
\hline$n=12$ & 0.990 & 0.985 & 0.979 & 0.969 \\
\hline
\end{tabular}

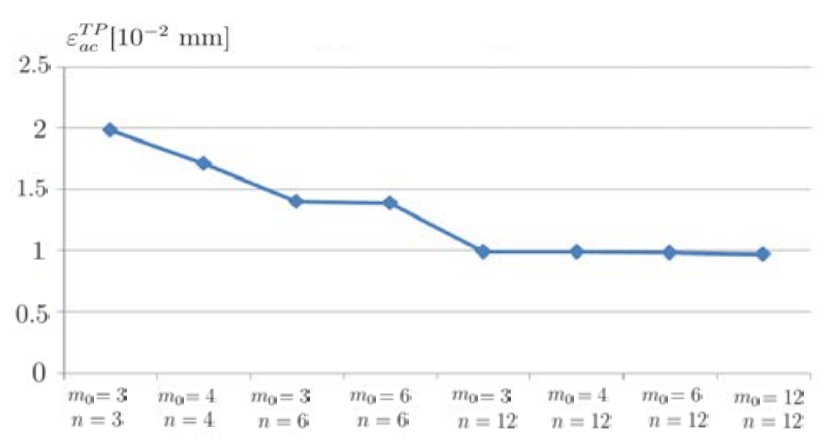

Fig. 5. $\varepsilon_{a c}^{T P}$ as a function of the number of measurement poses, $m_{0}$, and the number of measurements, $n$.

To minimize the effect of measurement errors on the robot end-effector pose error evaluation after error compensation, we should find optimal measurement configurations $\mathbf{q}_{i}^{\text {opt }}=\left[\begin{array}{lllllll}r_{1_{i}}^{\text {opt }} & \theta_{2_{i}}^{\text {opt }} & \theta_{3_{i}}^{\text {opt }} & \theta_{4_{i}}^{\text {opt }} & \theta_{5_{i}}^{\text {opt }} & \theta_{6_{i}}^{\text {opt }} & \theta_{7_{i}}^{\text {op }}\end{array}\right]^{T}$ of the KR500 robot and find the optimal position of the base attachment point $P_{a}$. The $z$-coordinate, $z_{p a}$, of point $P_{a}$ expressed in the base frame $\mathcal{F}_{b}$, is equal to $-0.891 \mathrm{~mm}$ due to the height of the rail. The optimisation of coordinate $y_{p_{a}}$ is redundant with the optimisation of joint length $r_{1}$ because this axis is assumed to be perfectly collinear with axis $y_{b}$. Therefore, $y_{p a}$ is set to $6 \mathrm{~m}$. As a consequence, the following optimisation is solved in order to find the optimum measurement poses and the optimum location of the base attachment point $P_{a}$ :

Thus, the optimisation problem can be formulated as follows

$$
\begin{array}{ll}
\min & \varepsilon_{a c}^{T P} \\
\text { over } & \mathbf{x}=\left[\mathbf{q}_{1}^{o p t}, \ldots, \mathbf{q}_{m_{0}}^{o p t}, x_{p_{a}}\right]^{T}
\end{array}
$$

subject to

$$
P b \quad\left\{\begin{array}{l}
0 \mathrm{~m} \leq r_{1_{i}}^{o p t} \leq 14 \mathrm{~m} \\
-70^{\circ} \leq \theta_{2_{i}}^{\text {opt }} \leq 70^{\circ} \\
z_{\theta_{7_{i}}^{\text {opt }} \geq 500 \mathrm{~mm}} \\
\|\mathbf{f}\|_{2}=4000 \mathrm{~N} \\
2 \mathrm{~m} \leq x_{p_{a}} \leq 6 \mathrm{~m}
\end{array}\right.
$$

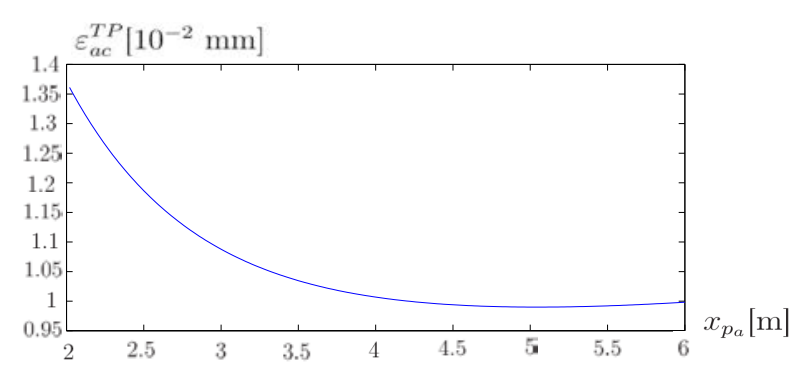

Fig. 6. $\quad \varepsilon_{a c}^{T P}$ as a function of $x_{p_{a}}$ for $n=12$ and $m_{0}=3$

where $m_{0}$ is the number of measurement poses.

In our case, the direction of the external force applied on the end-effector is defined by the position of the attachment point and the position of the robot end-effector. In the experimental setup the direction of external forces is obtained from the locations of points $P_{l_{2}}$ and $P_{l_{1}}$ located at the both ends of the spring (as shown in Fig. 2). Thus, the direction of external forces is expressed as follows :

$$
\mathbf{u}=\frac{P_{l_{2}}-P_{l_{1}}}{\left\|P_{l_{2}}-P_{l_{1}}\right\|_{2}}
$$

The external force is expressed as:

$$
\mathbf{f}=\|\mathbf{f}\| \cdot \mathbf{u}
$$

\section{Optimisation results}

The optimisation problem has been launched with a genetic algorithm for a single test-pose and for several measurement poses. It is noteworthy that the measurements can be repeated in the measurement poses in order to ease the experimentations.

The test-pose is defined by the following robot joint coordinate vector $\mathbf{q}_{T P}$ and the force $\mathbf{f}_{T P}$ applied on the robot end-effector

$$
\begin{aligned}
\mathbf{q}_{T P} & =\left[\begin{array}{llll}
8.5 m-10^{\circ} 35^{\circ} 39^{\circ} 81^{\circ} 22^{\circ}-10^{\circ}
\end{array}\right]^{T} \\
\mathbf{f}_{T P} & =\left[\begin{array}{ll}
-124.5 \mathrm{~N}-175.4 \mathrm{~N}-209.1 \mathrm{~N}
\end{array}\right]^{T}
\end{aligned}
$$

Table II gives the values of criterion $\varepsilon_{a c}^{T P}$ as a function of measurement poses and number of experiment. Figure 5 illustrates the value of $\varepsilon_{a c}^{T P}$ as a function of the number of measurement poses, $m_{0}$, and the the number of measurements, $n$. It is apparent that $\varepsilon_{a c}^{T P}$ is a minimum when $n=12$ and $m_{0}=12$. To improve the time required to achieve the stiffness identification for a same number of experiments, a minimum number of measurement configurations is needed. As a conclusion in order to minimize the number of robot configurations to make the experiments, twelve experiments will be performed with three measurement poses. It means that four measurements are made in each measurement pose.

Equations (14) to (16) give the joint angles vectors associated with the three optimal measurement poses.

$\mathbf{q}_{1}^{\text {opt }}=\left[5.3 \mathrm{~m}-37.3^{\circ} 57.6^{\circ} 80.8^{\circ} 101.2^{\circ}-27^{\circ}-82.2^{\circ}\right]^{T}(14)$

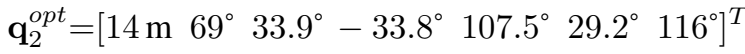

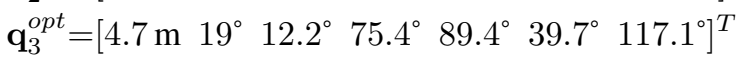


TABLE III

NOMINAL JOINT STIFFNESS VALUES

\begin{tabular}{c|cccccc}
\hline \hline$[\mathrm{N} / \mathrm{m}]$ & \multicolumn{6}{|c}{$[\mathrm{N} \mathrm{m} / \mathrm{rad}]$} \\
\hline$k_{r_{1}}$ & $k_{\theta_{2}}$ & $k_{\theta_{3}}$ & $k_{\theta_{4}}$ & $k_{\theta_{5}}$ & $k_{\theta_{6}}$ & $k_{\theta_{7}}$ \\
\hline $8.10^{7}$ & $3.8 .10^{6}$ & $6.6 .10^{6}$ & $3.9 .10^{6}$ & $5.6 .10^{5}$ & $6.6 .10^{5}$ & $4.7 .10^{5}$ \\
\hline \hline
\end{tabular}

Equation (17) gives the optimal Cartesian coordinates of the base attachment point $P_{a}$ expressed in the base frame $\mathcal{F}_{b}$.

$$
x_{p_{a}}=5.04 \mathrm{~m}, y_{p_{a}}=6 \mathrm{~m}, z_{p_{a}}=-0.891 \mathrm{~m}
$$

Fig. 6 presents value of the criterion $\varepsilon_{a c}^{T P}$ as a function of $x_{p_{a}}$ for twelve experiments and three measurement poses.

\section{SIMULATION}

To simulate the stiffness identification we consider the measurement poses defined by Eqs. (14) to (17) for the testpose defined by Eq. (12) and (13). This section aims to show the impact of the stiffness identification on the estimation of the robot end-effector deflection around the defined test-pose and for poses defined in the global workspace.

\section{A. Evaluation of the joint stiffness values}

The joint stiffness identification is performed by using the experimental setup presented in Section II. It is assumed that the geometric calibration has been realized. The spring scale is used to measure the magnitude of the external forces. Thus, to obtain the direction of external forces $\mathbf{f}$, a set of two SMR (Spherical Mounted Retro-reflector) are placed at the ends of the Spring scale at points $P_{l_{1}}$ and $P_{l_{2}}$. To measure the displacement of the robot end-effector, three SMR are set above $\left(P_{1}, P_{2}\right.$ and $\left.P_{3}\right)$.A measurement error is considered for each measurement. Moreover, the measurement system is a Leica Laser Tracker. Measurement errors are taken in a spherical coordinate system. Angles $\theta$ and $\phi$ are respectively taken in the intervals $[0, \pi / 2]$ and $[-\pi, \pi]$ with a uniform distribution, the radius $r$ follows a normal distribution.

$$
r \sim \mathcal{N}(\mu, \sigma)
$$

with $\mu=0$ and $\sigma=0.03 \mathrm{~mm}$. Eq. (6) expressed the displacement of the end effector as a function of the vector of nominal joint compliances presented in Table III and measurement errors. This equation can be computed as follows to take into account the number of measurement points, $p$, and the number of optimal measurement poses, $m_{0}$ :

$$
\mathbf{B}=\left[\begin{array}{c}
\mathbf{A}_{1}^{1} \\
\vdots \\
\mathbf{A}_{i}^{j} \\
\vdots \\
\mathbf{A}_{m_{0}}^{p}
\end{array}\right] \quad i=1, \ldots, m_{0}
$$

TABLE IV

IDENTIFIED JOINT STIFFNESS VALUES

\begin{tabular}{c|ccc|ccc}
\hline \hline \multirow{2}{*}{$\begin{array}{c}\text { Joint } \\
\text { number }\end{array}$} & \multicolumn{3}{|c|}{ Standard deviation } & \multicolumn{3}{c}{ Bias } \\
\cline { 2 - 7 } & $\sigma_{\mathbf{k}_{q_{e}} \cdot\left[10^{6}\right]}$ & unit & $\%$ & $b_{\mathbf{k}_{q_{e}} \cdot\left[10^{5}\right]}$ & unit & $\%$ \\
\hline 1 & 1.5831 & $\mathrm{~N} / \mathrm{m}$ & 1.979 & 2.474 & $\mathrm{~N} / \mathrm{m}$ & 0.309 \\
\hline 2 & 0.0027 & $\mathrm{~N} \mathrm{~m} / \mathrm{rad}$ & 0.072 & -0.003 & $\mathrm{~N} \mathrm{~m} / \mathrm{rad}$ & 0.008 \\
\hline 3 & 0.0245 & $\mathrm{~N} \mathrm{~m} / \mathrm{rad}$ & 0.371 & 0.0008 & $\mathrm{~N} \mathrm{~m} / \mathrm{rad}$ & 0.001 \\
\hline 4 & 0.0150 & $\mathrm{~N} \mathrm{~m} / \mathrm{rad}$ & 0.383 & -0.0192 & $\mathrm{~N} \mathrm{~m} / \mathrm{rad}$ & 0.049 \\
\hline 5 & 0.0367 & $\mathrm{~N} \mathrm{~m} / \mathrm{rad}$ & 6.554 & 0.0174 & $\mathrm{~N} \mathrm{~m} / \mathrm{rad}$ & 0.311 \\
\hline 6 & 0.0053 & $\mathrm{~N} \mathrm{~m} / \mathrm{rad}$ & 0.799 & 0.0008 & $\mathrm{~N} \mathrm{~m} / \mathrm{rad}$ & 0.012 \\
\hline 7 & 0.0290 & $\mathrm{~N} \mathrm{~m} / \mathrm{rad}$ & 6.172 & 0.0201 & $\mathrm{~N} \mathrm{~m} / \mathrm{rad}$ & 0.428 \\
\hline \hline
\end{tabular}

and

$$
\mathbf{c}=\left[\begin{array}{c}
\delta \mathbf{p}_{1}^{1} \\
\vdots \\
\delta \mathbf{p}_{i}^{j} \\
\vdots \\
\delta \mathbf{p}_{m_{0}}^{p}
\end{array}\right] \quad \quad \quad \quad j=1, \ldots, m_{0}
$$

In that case, the estimates value of joint compliances $\mathbf{x}_{q_{e}}$ can be obtained with the generalized inverse of $\mathbf{B}$ as follows:

$$
\mathbf{x}_{q_{e_{r}}}=\left(\mathbf{B}_{r}^{T} \mathbf{B}_{r}\right)^{-1} \mathbf{B}_{r}^{T} \mathbf{c}_{r} \quad r=1, \ldots, 200
$$

where $r$ is the number of repetitions of measurements.

Table IV presents the standard deviation, $\sigma_{\mathbf{k}_{q_{e}}}$, and the bias, $b_{\mathbf{k}_{q_{e}}}$, for the joint stiffness identification performed 200 times, the percentage of estimation error is also expressed in the Table IV to highlight the joints that are mainly responsible for the stiffness measurement inaccuracy.

These results are used further to evaluate the impact of the stiffness identification results on the estimation of the endeffector displacement in the global workspace and around the test-pose when an external wrench is applied on it.

\section{B. Estimation of the robot end-effector displacement}

Repetitive machining operations do not necessarily require a high accuracy in terms of stiffness identification. However, a correct estimation of the robot deflection is essential. The criterion illustrated hereafter aims to represent the accuracy of the estimation of the robot end-effector displacement. Thus, the smaller $\mu_{\mathbf{p}_{i}}^{j}$ represent the better displacement compensation.

$$
\mu_{\mathbf{p}_{i}}^{j}=\frac{\left\|\delta \mathbf{p}_{n i}^{j}-\delta \mathbf{p}_{e i}^{j}\right\|_{2}}{\left\|\delta \mathbf{p}_{n i}^{j}\right\|_{2}} \quad \begin{gathered}
i=1,2,3 \\
j=1, \ldots, 1000
\end{gathered}
$$

where $j$ is the number of validation measurement, $i$ is the number of measurement point, $\delta \mathbf{p}_{n i}^{j}$ is the nominal displacement of the robot end-effector, which is calculated with the nominal stiffness values presented in Table III. $\delta \mathbf{p}_{e i}^{j}$ is the estimation of the robot end-effector displacement calculated with the joint stiffness values given in Table IV. The joint stiffness estimation is computed as:

$$
\mathbf{k}_{q_{e}} \sim \mathcal{N}\left(\mu \mathbf{k}_{q_{e}}, \sigma \mathbf{k}_{q_{e}}\right)
$$


TABLE V

VALIDATION MEASUREMENTS

\begin{tabular}{c|c|c}
\hline \hline & \multicolumn{2}{|c}{$\mu_{\mathbf{p}_{i}}\left[10^{-3}\right]$} \\
\hline$i$ & Test-poses measurement & Global workspace measurement \\
\hline 1 & 1.6 & 6.8 \\
\hline 2 & 1.9 & 6.8 \\
\hline 3 & 2.0 & 6.8 \\
\hline \hline
\end{tabular}

where $\mu_{\mathbf{k}_{q_{e}}}$ is the average value of $\mathbf{k}_{q_{e}}$ and $\sigma_{\mathbf{k}_{q_{e}}}$ is the standard deviation of $\mathbf{k}_{q_{e}}$.

Moreover, the compensation of the end-effector pose error should be better around the test-pose defined in Eqs. (12) and (13).

Accordingly, a simulation is performed with a set of 1000 validation measurement configurations taken around the test-pose, and an other set of 1000 validation measurement configurations taken randomly in the global workspace of the KR500 mounted on a rail. For each measurement, the displacement of the robot end-effector is measured at points $P_{1}, P_{2}$ and $P_{3}$. Then, the criterion $\mu_{\mathbf{p}_{i}^{j}}$ is calculated with respect to the nominal displacement $\delta \mathbf{p}_{n i}^{j}$. Table $\mathrm{V}$ provides the results of those simulations.

It is apparent that the estimation of the robot end-effector displacement is identified with an accuracy of $0.16 \%$ to $0.2 \%$ around the test-pose and with an accuracy of $0.68 \%$ in the global workspace. Note that only the effect of the measurement errors on the evaluation of the robot endeffector pose error, while subjected to external wrenches, has been considered in the scope of this paper.

\section{CONCLUSION}

This paper dealt with a methodology to identify all stiffness parameters of an industrial serial robot mounted on a rail. Moreover, an existing test-pose based approach was used to identify the joint stiffness values of the KUKA KR500 MT robot mounted a rail, while minimizing the effect of measurement errors on the end-effector pose error evaluation after compensation. This method provides optimal measurement configurations adapted to the experimental setup.

In order to obtain the optimal measurement configurations, we firstly had to define the robot kinematic model. Then, we used the optimisation measurement configurations to run the simulations. Outcomes show the interest of using the optimisation method with defined machining configurations, called test pose.

In future work, the proposed methodology will be improved to consider the effect of the masses of the bodies on the robot deflection and the geometric errors. Moreover, a enhanced stiffness model of rail will be developed and some experimental validations will be performed in order to identify the geometric and stiffness parameters of the KUKA KR 500 MT robot mounted on a rail shown in Fig. 1.

\section{ACKNOWLEDGMENT}

This study is part of the ROBOFIN project managed by IRT Jules Verne (French Institute in Research and Technology in Advanced Manufacturing Technologies for Composite, Metallic and Hybrid Structures). The authors wish to associate the industrial and academic partners of this project; respectively DAHER, DCNS, STX France, Loiretech, Multiplast, Europe Technologies, Coriolis Composites, IRCCyN (UMR CNRS-ECN-Nantes University 6597).

\section{REFERENCES}

[1] W. Khalil and E. Dombre, Modeling, identification and control of robots. Butterworth-Heinemann, 2004.

[2] E. Abele, M. Weigold, and S. Rothenbücher, "Modeling and identification of an industrial robot for machining applications," CIRP AnnalsManufacturing Technology, vol. 56, no. 1, pp. 387-390, 2007.

[3] G. Alici and B. Shirinzadeh, "Enhanced stiffness modeling, identification and characterization for robot manipulators," Robotics, IEEE Transactions on, vol. 21, no. 4, pp. 554-564, 2005.

[4] A. Nubiola and I. Bonev, "Absolute calibration of an ABB IRB 1600 robot using a laser tracker," Robotics and Computer-Integrated Manufacturing, 2012.

[5] C. Dumas, S. Caro, S. Garnier, and B. Furet, "Joint stiffness identification of six-revolute industrial serial robots," Robotics and ComputerIntegrated Manufacturing, vol. 27, no. 4, pp. 881-888, 2011.

[6] M. Gautier, A. Jubien, A. Janot, and P.-P. Robet, "Dynamic identification of flexible joint manipulators with an efficient closed loop output error method based on motor torque output data," in Robotics and Automation (ICRA), 2013 IEEE International Conference on. IEEE, 2013, pp. 2949-2955.

[7] D. S. Milutinovic, B. M. Kokotovic, M. M. Glavonjic, S. T. Zivanovic, and K. F. Ehmann, "Cartesian compliance identification and analysis of an articulated machining robot," FME Transactions, vol. 41, pp. $83-95,2013$

[8] C. Lightcap, S. Hamner, T. Schmitz, and S. Banks, "Improved positioning accuracy of the pa10-6ce robot with geometric and flexibility calibration," Robotics, IEEE Transactions on, vol. 24, no. 2, pp. 452456, 2008.

[9] Z. Pan, H. Zhang, Z. Zhu, and J. Wang, "Chatter analysis of robotic machining process," Journal of materials processing technology, vol. 173, no. 3, pp. 301-309, 2006.

[10] C. Gong, J. Yuan, and J. Ni, "Nongeometric error identification and compensation for robotic system by inverse calibration," International Journal of Machine Tools and Manufacture, vol. 40, no. 14, pp. 2119 2137,2000

[11] D. Daney, Y. Papegay, and B. Madeline, "Choosing measurement poses for robot calibration with the local convergence method and tabu search," The International Journal of Robotics Research, vol. 24, no. 6, pp. 501-518, 2005.

[12] H. Zhuang, K. Wang, and Z. S. Roth, "Optimal selection of measurement configurations for robot calibration using simulated annealing," in Robotics and Automation, 1994. Proceedings., 1994 IEEE International Conference on. IEEE, 1994, pp. 393-398.

[13] C. Dumas, S. Caro, M. Cherif, S. Garnier, B. Furet et al., "Joint stiffness identification of industrial serial robots," Robotica, vol. 30 , no. 4, pp. 649-659, 2012.

[14] A. Klimchik, Y. Wu, A. Pashkevich, S. Caro, and B. Furet, "Optimal selection of measurement configurations for stiffness model calibration of anthropomorphic manipulators," Applied Mechanics and Materials, vol. 162, pp. 161-170, 2012.

[15] Y. Wu, A. Klimchik, A. Pashkevich, S. Caro, and B. Furet, "Optimality criteria for measurement poses selection in calibration of robot stiffness parameters," in Proceedings of the ASME 2012 11th Biennial Conference on Engineering Systems Design and Analysis, 2012, pp. $1-10$.

[16] X. Zhang, W. Yang, X. Cheng, and Y. Chen, "Stiffness identification for serial robot manipulator based on uncertainty approach," Intelligent Robotics and Applications, pp. 378-388, 2011.

[17] A. Klimchik, A. Pashkevich, Y. Wu, S. Caro, B. Furet et al., "Design of calibration experiments for identification of manipulator elastostatic parameters," Journal of Mechanics Engineering and Automation, vol. 2(9), pp. 531-542, 2012. 